\title{
An enhanced MPR-based solution for flooding of broadcast messages in OLSR wireless ad hoc networks
}

\author{
Tzu-Hua Lin ${ }^{\mathrm{a}}$, Han-Chieh Chao ${ }^{\mathrm{a}, \mathrm{b}, *}$ and Isaac Woungang ${ }^{\mathrm{c}}$ \\ ${ }^{a}$ Department of Electrical Engineering, National Dong Hwa University, Hualien, Taiwan \\ ${ }^{\mathrm{b}}$ Institute of Computer Science \& Information Engineering, and Department of Electronic Engineering, \\ National Ilan University, I-Lan, Taiwan \\ ${ }^{\mathrm{c}}$ Department of Computer Science, Ryerson University, Toronto, Canada
}

\begin{abstract}
In an Optimized Link State Routing (OLSR)-based mobile wireless network, optimizing the flooding of broadcast messages is a challenging task due to node's mobility and bandwidth resource consumption. To complement existing solutions to this problem, the Multi-Point Relays (MPR) selection has recently been advocated as a promising technique that has an additional feature of reducing the number of redundant re-transmission occurring in the network. This paper continuous on the investigation of an existing MPR-based solution, arguing that by considering a cost factor as an additional decision parameter in selecting the MPR nodes, the enhanced MPR selection algorithm leads to less packet loss in the network. Simulation experiments are presented to validate the stated goal, using the average packet loss ratio as the performance metric.
\end{abstract}

Keywords: Mobile wireless ad hoc network, link stability, OLSR, proactive routing, flooding, MPR selection

\section{Introduction}

With the widespread availability and rapid evolution of wireless local area network technologies such as 802.11 [8], Bluetooth [6], to name a few, the use of MANETs is growing fast [9-11]. In a MANET, there is no need for an infrastructure or a centralized administration for nodes to communicate with each other. Nodes cooperate to provide connectivity and services. However, this flexibility is accompanied by several challenges, in particular, from a routing prospective. In MANETs, the bandwidth, power, speed of mobile nodes, density of the topology, distribution and location of mobile nodes, are among the various factors that can influence the routing process [3,12], hereby the establishment of a routing path between a node pair. These factors, if not well controlled, can lead to an increasing packet loss and a decreasing network performance and link stability. As far as routing is concerned, one of the primary challenges in a MANET is to determine methods for reducing the waste of bandwidth and power consumption while ensuring a stable transmission of information, and quickly responding to network

\footnotetext{
${ }^{*}$ Corresponding author: Han-Chieh Chao, Institute of Computer Science and Information Engineering, National I-lan University, Sec. 1, Shen-Lung Rd., I-Lan, Taiwan, Republic of China. Tel.: +886 39357400 - x.251; Fax: +866 39354238 ; E-mail: hcc@niu.edu.tw.
} 
changes. Of course, these methods depend on the capability of optimizing broadcast messages in the network.

To cope with this issue, one of the recent proactive protocols known as OLSR [13] has been advocated as a promising routing protocol for multi-hop wireless ad-hoc networks. In OLSR, only a subset of pre-selected nodes referred to as MPR nodes have specific functionalities of performing topological advertisements as well as broadcasting and forwarding of control messages, with the goal to reduce the impact of message flooding and control overhead. The set of MPR nodes is chosen in such a way that a minimum of one-hop symmetric neighbors is able to reach all the symmetric two-hop neighbors. To calculate the MPR set, the node must possess the knowledge of the link state information about all one-hop and two-hop neighbors. The objective of the MPR technique is to reduce the number of redundant retransmissions, while ensuring reliable delivery of broadcast messages. In a MPR-based OLSR wireless ad hoc network, every node needs to issue periodic Hello message to update the 1-hop neighbors set and 2-hop neighbors set tables. Every node must dynamically select a neighbor node as MPR node at a time. After this selection is completed, messages are broadcasted, and control messages as well as traffic data that all packets should transmit through MPR nodes are identified.

This paper continues on the investigation of a heuristic introduced in [4] (our so-called MPR-based OLSR heuristic) for the selection of MPR nodes. We consider a cost factor [4] as an additional decision parameter within the MPR-based OLSR heuristic, and we demonstrate by simulation that the resulting heuristic (so-called Enhanced MPR-based OLSR heuristic - here denoted as EMPR-based OLSR) has superior performance over MPR-OLSR in terms of average packet loss ratio in the network. Our primary objective is to investigate the impact of packet loss during transmission when the coverage of a node's selected MPR increases due to the introduction of the above-mentioned additional cost factor in the computation of the MPR selection node.

The paper is organized as follows. Section 2 presents the related works. Section 3 overviews the MPR selection concept. Section 4 describes the proposed EMPR-based OLSR heuristic and contrasts it against the MPR-based OLSR approach. In Section 5, simulation results comparing the proposed EMPR-based heuristic against the MPR-based heuristic are presented. To this effect, OPNET [14] is used as the simulation tool, and the packet loss is considered as the performance metric. Finally, in Section 6, some concluding remarks are given.

\section{Related work}

The selection of the MPR set of nodes is a fundamental operation in the OLSR protocol. Most solutions to this problem are in the form of heuristics. Representative ones deal with MPR-based flooding techniques $[2,4,15,16]$, as well as reducing the number of collisions or maximizing the bandwidth [7, 17]. With respect to link stability, the authors in [18] studied the link lifetime using various mobility models. They derived a formula to calculate the expected value of each node in order to select high expected value of MPR. Similarly, in [5], some statistical techniques based on link stability metrics were used for selecting MPR nodes while enhancing the route reliability and decreasing the packet loss. Other OLSR enhancements deal with quality of service routing based MPR selection, by combining the MPR selection mechanism with the path determination algorithm [19-21]. In almost all these representative solutions, the goal has been to determine the impact the selection of MPRs has on the performance of OLSR

This paper introduces an additional parameter in the MPR selection method proposed in [4], namely the cost values calculation by all 1-hop and 2-hop neighbor nodes. Our goal is to improve the process of MPR 


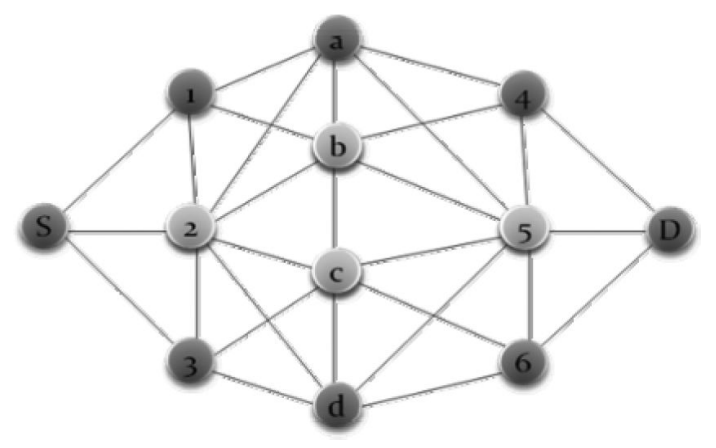

Fig. 1. Example network.

through reducing the total packet loss during transmission, thereby, achieving a superior performance than that of the MPR-based OLSR heuristic.

\section{Overview of MPR selection}

The OLSR protocol has been extensively investigated in the literature. Rather than re-describing its basic functionality, this Section is meant to setup the context of the study carried in this paper. OLSR is an optimization of the pure link state-based routing protocol [13]. Two important concepts were introduced in the RFC3626 OLSR draft [23] that can enable OLSR extensions, namely, neighbor discovery and Multipoint-Relay selection (MPR). This paper focuses on using MPR. The later is particularly important since it has an immediate effect on the routing protocol's performance. Indeed, the overhead of control traffic generated by the OLSR protocol as well as the flooding efficiency depends on the MPR selection, which itself, is known as a NP-hard problem [17]. In the MPR-based OLSR protocol, each node selects a subset of its one-hop neighbors as its MPR list, based on periodic HELLO messages received by these neighborhood nodes (one-hop neighbors and two-hop neighbors). The compilation of MPR lists then builds a database of nodes that are used in the routing process to compute the shortest paths to all possible destinations in the network. Compared to classical link-state algorithms, MPR-based OLSR algorithms have been proved to significantly reduce the number of message re-transmissions and control traffic [1, $2,4,7,16,18,19,22-24]$, to name a few.

This paper continues the investigation of the MPR-based OLSR heuristic proposed in [4]. One of the drawbacks of this heuristic is that in the employed MPR technique, only a selected number of nodes propagate the message, which might limit the chances that the message reaches all the nodes in the network. The situation is even worse when the bit error rate in the radio transmission is high $[4,13]$, leading to dramatic packets loss in the network. The MPR-based OLSR heuristic [4] proceeds in three main steps (as detailed in the next section) among which the second one is devoted to the optimization of the MPR set. An analysis of this MPR-based OLSR heuristic has shown that it is within a $\log n$ factor from optimality [4].

Our enhancement to the above MPR-based OLSR (so-called EMPR-based OLSR) consists of keeping the core of the MPR-based OLSR heuristic, but introducing a cost factor (in the above-mentioned Step 2 ) as an additional decision parameter when designing the optimized MPR set.

The EMPR-based OLSR heuristic produces a larger cover range for the MPR set compared to that of the MPR-based OLSR heuristic. As an example, the MPR-based OLSR heuristic applied to the example network shown in Fig. 1 produces $\{2, \mathrm{~b}, \mathrm{c}, 5\}$ as optimized MPR set with a cover range as depicted in 


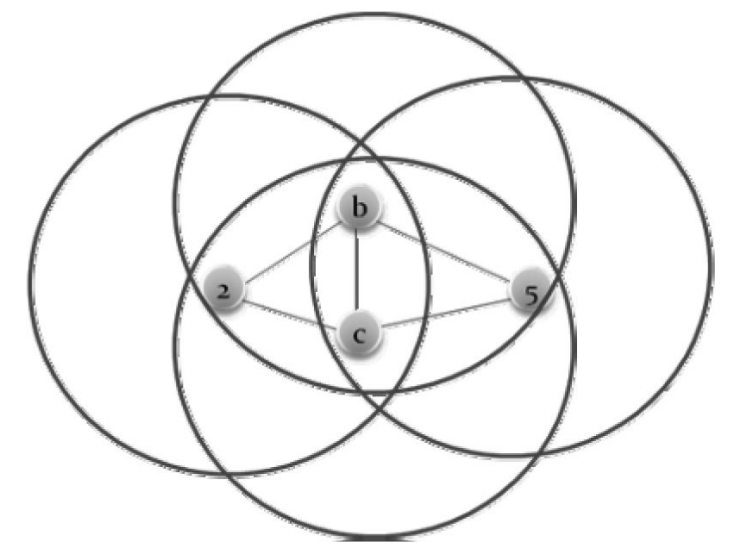

Fig. 2. MPR set cover range when using the MPR-based OLSR heuristic.

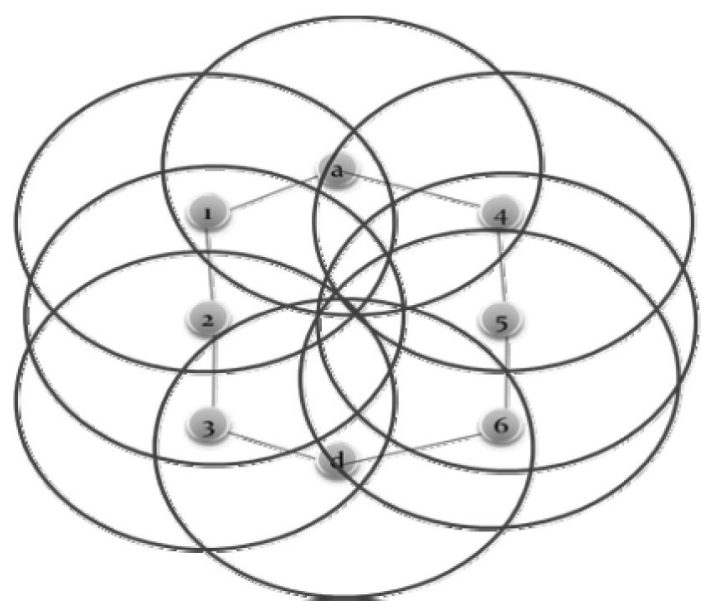

Fig. 3. MPR set cover range when using the EMPR-based OLSR heuristic.

Fig. 2, whereas for the same network, our EMPR-based OLSR heuristic yields $\{1,2,3$, a, d, 4, 5, 6$\}$ as optimized MPR set with a cover range as depicted in Fig. 3.

Our goal is to demonstrate that the EMPR-based OLSR heuristic outperforms the MPR-based OLSR heuristic in terms of packets loss reduction in the network. In the sequel, we adopt the same notations that were used in [4] and we describe the MPR-based OLSR and the EMPR-based OLSR heuristics.

\section{Enhanced MPR-based OLSR}

The network is represented as a graph $\mathrm{G}$ (i.e. a set $\mathrm{V}$ of nodes and a set of neighbors for each node). For each element $x$ in $V, M(x)$ is the neighborhood of $x, N(x)$ is the set of one-hop neighbors of $x$ (i.e. $x$ covers any element of $N(x)$ ), $N^{2}(x)$ is the set of two-hop neighbors of $x, D(x)$ is the degree of $x$ (i.e. the number of links connected to $\mathrm{x}$ ), and MPR(x) is the selected MPR set of node $\mathrm{x}$.

The MPR-based OLSR heuristic as introduced in [9] can be summarized as follows:

- Step 1: Start with an empty MPR set MPR(x) 
- Step 2: Calculate $\mathrm{N}(\mathrm{x}), \mathrm{N}^{2}(\mathrm{x})$ and $\mathrm{D}(\mathrm{x})$ for all $\mathrm{x}$ in $\mathrm{N}(\mathrm{x})$

- Step 3: Select as MPR nodes those nodes in $\mathrm{N}(\mathrm{x})$ which are the only neighbor of some node in $\mathrm{N}^{2}(\mathrm{x})$

- Step 4: As long as there exist some node in $\mathrm{N}^{2}(\mathrm{x})$ which is not covered by the set MPR(x), do the following:

* For each node $\mathrm{z}$ in $\mathrm{N}(\mathrm{x})$ which is not element of MPR(x), determine the number of nodes that $\mathrm{z}$ covers among uncovered nodes in $\mathrm{N}^{2}(\mathrm{x})$.

* Add in MPR( $\mathrm{x}$ ) the node $\mathrm{z}$ for which this number is maximum

Let $S_{1}$ be the set of nodes selected in Step 3 and $\left(x_{1}, \ldots, x_{k}\right)$ be the set of nodes selected in the abovementioned Step 3 and Step 4 of MPR-based OLSR heuristic respectively. Let $\mathrm{N}_{1}^{2}$ be the set of nodes in $\mathrm{N}^{2}(\mathrm{~s})$ that are neighbors of some nodes $\mathrm{s}$ in $\mathrm{S}_{1}$. Let $\mathrm{N}^{2}=\mathrm{N}^{2}(\mathrm{~s})-\mathrm{N}_{1}^{2}$. A unit cost Cy, associated with each node $\mathrm{y}$ in $\mathrm{N}^{2}$, is defined as follows [8]:

$$
c_{y}=\frac{1}{\left|N^{\prime}\left(x_{j}\right)-\cup_{j=1}^{j-1} N^{\prime}\left(x_{j}\right)\right|}
$$

where $|\mathrm{P}|$ denotes the cardinality of the set P. As pointed out in [4], for each $\mathrm{x}_{t}$ chosen by the MPR-based OLSR algorithm, this unit cost is equally distributed among nodes that are newly covered in $\mathrm{N}^{2}$. Our proposed EMPR-based OLSR heuristic takes advantage of this feature. Its stepwise description follows.

- Step 1: Same as in the MPR-based OLSR heuristic

- Step 2: Calculate $\mathrm{N}(\mathrm{x}), \mathrm{N}^{2}(\mathrm{x})$ and $\mathrm{D}(\mathrm{x})$ for all $\mathrm{x}$ in $\mathrm{N}(\mathrm{x})$. Calculate the cost value $\mathrm{C}_{z}$ associated to each node $\mathrm{z}$ in $\mathrm{N}(\mathrm{x})$ using Eq. (1)

- Step 3: Select as MPR nodes those nodes in $\mathrm{N}(\mathrm{x})$ which are the only neighbor of some node in $\mathrm{N}^{2}(\mathrm{x})$. Enlarge the MPR(x) set by adding those nodes in $\mathrm{N}(\mathrm{x})$ that have the lowest cost values (there might be only one such node or many such nodes)

- Step 4: As long as there exists some node in $\mathrm{N}^{2}(\mathrm{x})$ which is not covered by the set MPR(x), do the following:

* For each node $\mathrm{z}$ in $\mathrm{N}(\mathrm{x})$ which is not element of MPR(x), determine the number of nodes that $\mathrm{z}$ covers among uncovered nodes in $\mathrm{N}^{2}(\mathrm{x})$.

* Add in MPR(x) the node $\mathrm{z}$ for which this number is maximum or add in MPR(x) the node $\mathrm{z}$ with lower cost value.

The distinction between the MPR-based OLSR heuristic and the EMPR-based heuristic relies in the introduction of the aforementioned cost value $\mathrm{C}_{z}$ associated with each node $\mathrm{z}$ in $\mathrm{N}(\mathrm{x})$, which contributes to the construction of the optimized MPR(x) set.

It should be noticed that the introduction of the cost value in the MPR selection calculation has resulted to the following impacts. The first one is an increase of the MPR count. In this case, Step 4 of the proposed EMPR heuristic will attempt to control this side effect by allowing each node to periodically update its neighbor table using "HELLO Messages", which would result in allowing each MPR selected node to flood TC message to all other MPR nodes in the network. The second one is an increase of the coverage of a node's selected MPRs, which in turn will increase the redundancy of TC-messages forwarding. In this case, the MPR-forwarding strategy described in Step 4 of our EMPR heuristic would handle the duplicate re-transmissions directly at the design level when selecting the MPR node set. Indeed, when a mobile node $\mathrm{n} 1$ will receive a packet, each node $\mathrm{n} 2$ would be required to determine whether node $\mathrm{n} 1$ has been selected as MPR node - note that this is realized through computing the node 
Table 1

Simulation Environment and Parameters setup

\begin{tabular}{cc}
\hline Parameter & Meaning \\
\hline Number of nodes & 50 \\
Communication range & 200 \\
Mobility model & Random waypoint \\
Wireless LAN & $802.11 \mathrm{~g}$ \\
IP protocol & IPv6 \\
Transmission type & G723 voice bps \\
Data rate & $11 \mathrm{Mbps}$ \\
Maximum received lifetime & $0.5 \mathrm{sec}$ \\
Simulation start time & $10 \mathrm{sec}$ \\
Channel match criterion & Strict match \\
Traffic duration & $3600 \mathrm{sec}$ \\
Traffic mix & All explicit \\
Traffic start time & $60 \mathrm{sec}$ \\
Simulation area & $2 * 2 \mathrm{~km}^{2}$ \\
\hline
\end{tabular}

$\mathrm{n} 2$ 's neighbor's table, the node n2's two-hop neighbor table and the number of neighbors of node $\mathrm{n} 2$. If the answer is yes, then node $\mathrm{n} 1$ will be sending the packets to all other designated neighbor nodes. Otherwise, node $n 1$ will not send the packets, but will remain active.

For both heuristics, experimenting the processes of MPR selection within a specific node using OPNET revealed that this additional decision parameter helps the EMPR-based OLSR heuristic producing a larger cover range for the MPR set compared to that generated by the MPR-based OLSR heuristic. This feature helps reducing the number of packets loss in the network during the transmission period (as illustrated through the simulation results shown in Section 5).

\section{Performance evaluation}

In this Section, we evaluate the performance of our EMPR-based OLSR heuristic against that of the MPR-based heuristic via simulations. The performance comparison metric is the average packet loss ratio, representing the ratio of packets lost to the total packets generated in a certain time period. The simulation tool is OPNET [14] and the location of the nodes is random. The simulation environment and parameters setup are captured in Table 1.

We have used a single seed value and have ran several simulations. For the same scenario, the simulation time was set to 1 hour and the node's speed was varied from $10 \mathrm{~m} / \mathrm{s}$ to $90 \mathrm{~m} / \mathrm{s}$.

Figure 4 depicts the speed of the mobile node (x axis) versus the amount of traffic it has received (y axis) in terms of number of bits. Figure 5 illustrates the MPR count time average versus the mobile node's speed when running both heuristics. Finally, Fig. 6 depicts the average packet loss ratio versus the mobile node's speed when running both heuristics. It is observed that the EMPR-based heuristic outperforms the MPR-based OLSR heuristic in terms of total number of packet loss during transmission.

\section{Concluding remarks}

In this paper, we have proposed an enhanced version (called EMPR-based OLSR heuristic) of an existing MPR-based OLSR selection method in MANETs (referred to as MPR-based OLSR heuristic). Our proposal considers the cost value as an additional factor of MPR selection. Our simulation results 


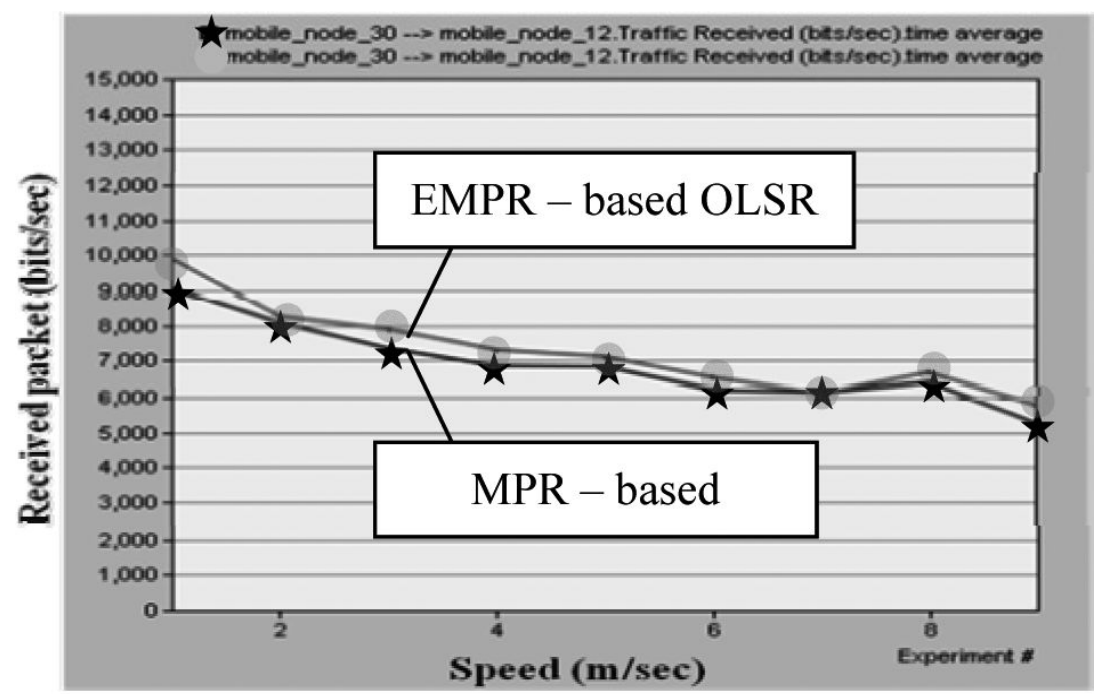

Ex: 1 denote $10 \mathrm{~m} / \mathrm{sec}_{\mathrm{r}} \underline{2}$ denote $20 \mathrm{~m} / \mathrm{sec}_{\mathrm{r}}$ and so on

Fig. 4. Mobile node's speed versus the amount of traffic received.

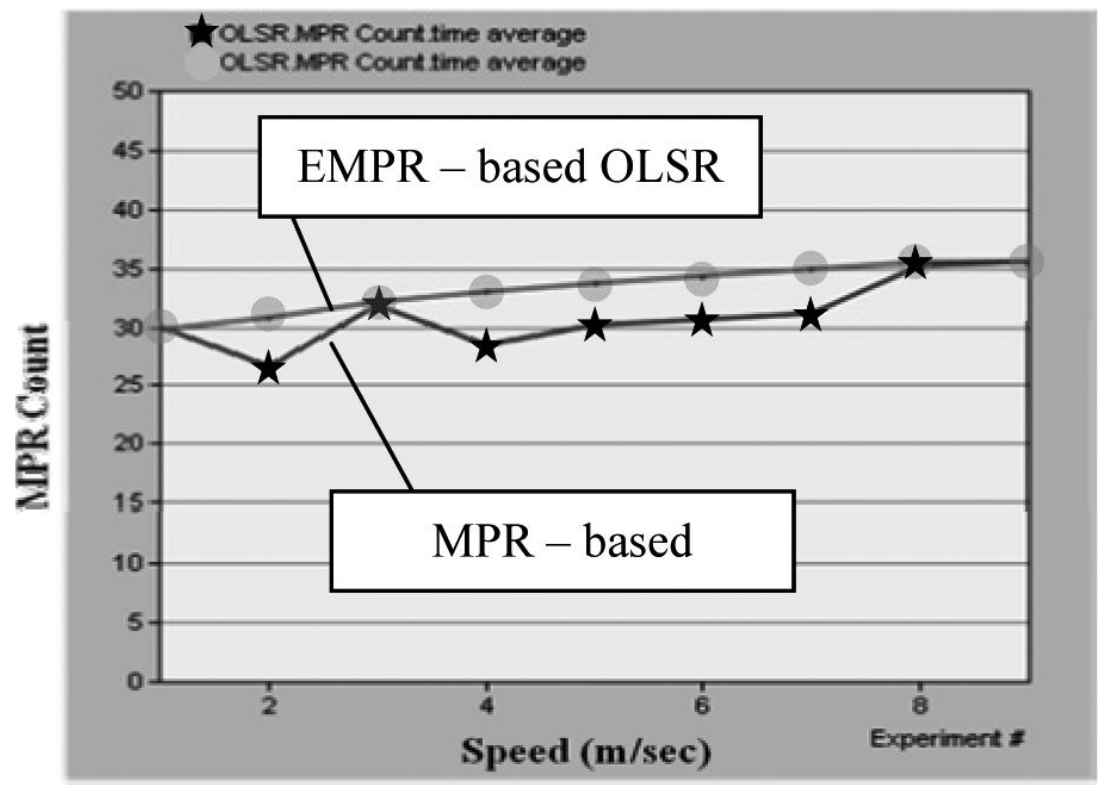

Ex: 1 denote $10 \mathrm{~m} / \mathrm{sec}_{1} 2$ denote $20 \mathrm{~m} / \mathrm{sec}_{1}$ and so on

Fig. 5. MPR count versus mobile node's speed.

have shown that the EMPR-based OLSR heuristic yields a better average packet loss ratio compared to the MPR-based OLSR heuristic. As future work, comparing the proposed EMPR-based OLSR heuristic against few other existing MPR-based selection methods for link stability in MANETs would be an interesting work. Also, we have not simulated the change in MPR coverage, nor investigating the NS_MPR forwarding TC message strategy. 


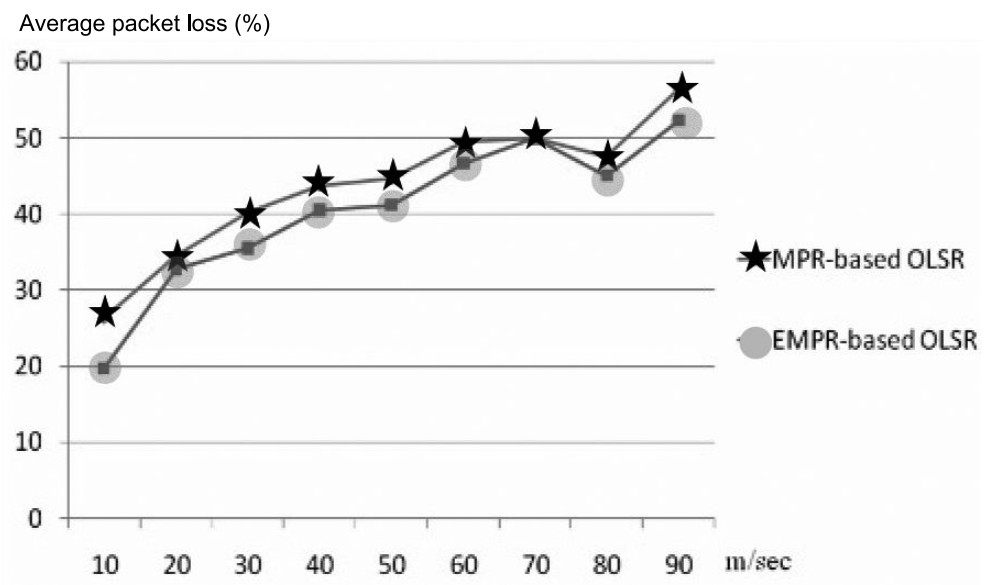

Fig. 6. Average packet loss versus the mobile node's speed.

\section{Acknowledgements}

This work is partially supported by National Science Council of Taiwan, under grant number NSC972219-E-197-001 and NSC97-2219-E-197-002.

\section{References}

[1] A. Benslimane, R. El Khoury, R. El Azouzi and S. Pierre, Energy Power-Aware Routing in OLSR Protocol, In Proc. 1st International Mobile Computing and Wireless Communication Conference, Sept. 2006, pp. 14-19.

[2] A. Laouiti, P. Muhlethaler, A. Najid and E. Plakoo, Simulation Results of the OLSR Routing Protocol for Wireless Network, MedHoc-Net, Sardegna, Italy, 2002.

[3] A.M. Hanashi, I. Awan and M.E. Woodward, Performance Evaluation with Different Mobility Models for Dynamic Probabilistic Flooding in MANETs, Mobile Information Systems 5(1) (2009), 65-80.

[4] A. Qayyum, L. Viennot and A. Laouiti, Multipoint Relaying for Flooding Broadcast Messages in Mobile Ad Hoc Networks, Proc. of 35th International Conference on System Sciences, Hawaii, USA, 2002.

[5] B. Mans and N. Shrestha, Performance Evaluation of Approximation Algorithms for Multipoint Relay Selection, MedHoc-Net04, Bodrum, Turkey, June 27-30, 2004

[6] Bluetooth Specifications, http://www.bluetooth.org/ (Last visited June 3, 2010).

[7] D. Gantsou, P. Sondi and S. Hanafi, Revisiting Multipoint Relay Selection in the Optimized Link State Routing Protocol, International Journal of Communication Networks and Distributed Systems, Inderscience, UK 2(1) (2009), 4-15.

[8] IEEE Standards, Standards.ieee.org/getieee802/802.11.html (Last visited June 3, 2010).

[9] S. Corson and J. Macker, Mobile Ad hoc Networking (MANET): Routing Protocol Performance Issues and Evaluation Considerations, RFC2501, Jan. 1999, http://www.faqs.org/rfcs/rfc2501.html (Last visited June 3, 2010).

[10] H. Zhang, H. Zhang, J. Guan and Y. Qin, A New Architecture of Multicast Interworking between MANET and Fixed Network, Journal of Internet Technology 8(1) (2007), 141-150.

[11] J. Chen, AMNP: ad hoc multichannel negotiation protocol with broadcast solutions for multi-hop mobile wireless networks, IET Communications 4(5) (2010), 521-531.

[12] V. Pham, E. Larsen, ØKure and P. Engelstad, Routing of internal MANET traffic over external networks, Mobile Information Systems 5(3) (2009), 291-311.

[13] T. Clausen, P. Jacquet, A. Laouiti, P. Muhlethaler, A. Qayyum and L. Viennot, Optimized Link State Routing Protocol, IEEE INMIC Pakistan, 2001.

[14] OPNET Simulator, http://www.opnet.com/ (Considerations, RFC2501, Jan. 1999, http://www.faqs.org/rfcs/rfc2501. html), (Last visited June 3, 2010).

[15] T. H. Clausen, G. Hansen, L. Christensen and G. Behrmann, The Optimized Link State Routing Protocol, Evaluation through Experiments and Simulation, IEEE Symposium on Wireless Personal Mobile Communications, Sept. 2001.

[16] P. Jacquet, A. Laouiti, P. Minet and L. Viennot, Performance of Multipoint Relaying in Ad Hoc Mobile Routing Protocols, Networking 2002, Pise, Italy, 2002. 
[17] P. Jacquet, A. Laouiti, P. Minet and L. Viennot, Performance Analysis of OLSR Multipoint Relay Flooding in Two Ad Hoc Wireless Network Models, Research Report-4260, INRIA, Sept. 2001, RSRCP Journal, Special Issue on Mobility and Internet.

[18] E. Baccelli and P. Jacquet, Flooding Techniques in Mobile Ad-Hoc Networks, Research Report RR-5002, INRIA, 2003.

[19] M. Gerharz, C. Waal, de, M. Frank and P. Martini, Link Stability in Mobile Wireless Ad Hoc Networks, In Proc. of the 27th Annual IEEE Conference on Local Computer Networks (LCN), Nov 6-8, Tampa, Florida, USA, pp. 30-39, 2002.

[20] S. Obilisetty, A. Jasti and R. Pendse, Link Stability Based Enhancements to OLSR (LS-OLSR), In Proc. of 62nd IEEE Vehicular Technology Conference (VTC-2005), Dallas, USA, Sept. 2005.

[21] N. Ghanem, S. Boumerdassi and E. Renault, New Energy Saving Mechanisms for Mobile Ad Hoc Networks using OLSR, In Proc. of the 2nd ACM Intl. Workshop on Performance Evaluation of Wireless Ad Hoc, Sensor, and Ubiquitous Networks, Oct. 2005, pp. 273-274.

[22] Z. Guo and B. Malakooti, Energy Aware Proactive MANET Routing with Prediction on Energy Consumption, In Proc. of the Intl. Conference on Wireless Algorithms, Systems and Applications, Aug. 2007, pp. 287-293.

[23] RFC3626 Draft, http://www.ietf.org/rfc/rfc3626.txt (Last visited June 3, 2010).

[24] M. Ikeda, L. Barolli, G. De Marco, T. Yang, A. Durresi and F. Xhafa, Tools for Performance Assessment of OLSR Protocol, Mobile Information Systems 5(2) (2009), 165-176.

Lin Tzu-Hua was born in Kaohsiung, Taiwan in 1984. He received his B.S. and M.S. degrees from the Department Electrical Engineering, Da-Yeh University and National Dong Hwa University in 2003 and 2009 respectively. Since then, he has been with the National Cheng Kung University, Tainan, Taiwan, working towards his Ph. D degree. His research interests include Mobile communication systems, Vehicle wireless communication, Cooperative networking, and Network coding.

Han-Chieh Chao is a joint appointed Full Professor of the Department of Electronic Engineering and Institute of Computer Science \& Information Engineering. He also serves as the Dean of the College of Electrical Engineering \& Computer Science for National Ilan University, I-Lan, Taiwan, R.O.C. He has been appointed as the Director of the Computer Center for Ministry of Education starting from September 2009 as well. His research interests include High Speed Networks, Wireless Networks, IPv6 based Networks, Digital Creative Arts and Digital Divide. He received his MS and Ph.D. degrees in Electrical Engineering from Purdue University in 1989 and 1993 respectively. He has authored or co-authored 4 books and has published about 200 refereed professional research papers. He has completed 80 MSEE thesis students and $2 \mathrm{PhD}$ students. Dr. Chao has received many research awards, including Purdue University SRC awards, and NSC research awards (National Science Council of Taiwan). He also received many funded research grants from NSC, Ministry of Education (MOE), RDEC, Industrial Technology of Research Institute, Institute of Information Industry and FarEasTone Telecommunications Lab. Dr. Chao has been invited frequently to give talks at national and international conferences and research organizations. Dr. Chao is also serving as an IPv6 Steering Committee member and co-chair of R\&D division of the NICI (National Information and Communication Initiative, a ministry level government agency which aims to integrate domestic IT and Telecom projects of Taiwan), Co-chair of the Technical Area for IPv6 Forum Taiwan, the executive editor of the Journal of Internet Technology and the Editor-in-Chief for International Journal of Internet Protocol Technology and International Journal of Ad Hoc and Ubiquitous Computing. Dr. Chao has served as the guest editors for Mobile Networking and Applications (ACM MONET), IEEE JSAC, IEEE Communications Magazine, Computer Communications, IEE Proceedings Communications, the Computer Journal, Telecommunication Systems, Wireless Personal Communications, and Wireless Communications \& Mobile Computing. Dr. Chao is an IEEE senior member and a Fellow of IET (IEE). He is a Chartered Fellow of British Computer Society.

Isaac Woungang received his M.S. and Ph. D degrees in Mathematics from the Universita $f$ de la Mafditerranafe-Aix Marseille II, France, and the Universita $f$ du Sud, Toulon et Var, France, in 1990 and 1994 respectively. In 1999, he received a M.S from the INRS-Materials and Telecommunications, University of Quebec, Montreal, Canada. From 1999 to 2002, he worked as a software engineer at Nortel Networks. Since 2002, he has been with Ryerson University, where he is an Associate professor of Computer Science. In 2004, he founded the Distributed Applications and Broadband NEtworks Laboratory (DABNEL) R\&D group. His research interest includes Network security, Computer communication networks, Mobile communication systems, computational intelligence applications in telecommunications, and Coding theory. Dr. Woungang serves as Editor-in-Chief of the International Journal of Communication Networks \& Distributed Systems (IJCNDS), Inderscience, U.K, and the International Journal of Information and Coding Theory (IJICoT), Inderscience, U.K, as Associate Editor of the International Journal of Communication Systems (IJCS), Wiley, and Associate Editor of Computer and Electrical Engineering, An International Journal (C\&EE), Elsevier. Dr. Woungang edited several books in the areas of wireless ad hoc networks, wireless sensor networks, wireless mesh networks, communication networks \& distributed systems, and information \& coding theory, published by reputed publishers such as Springer, Elsevier, and World Scientific. 

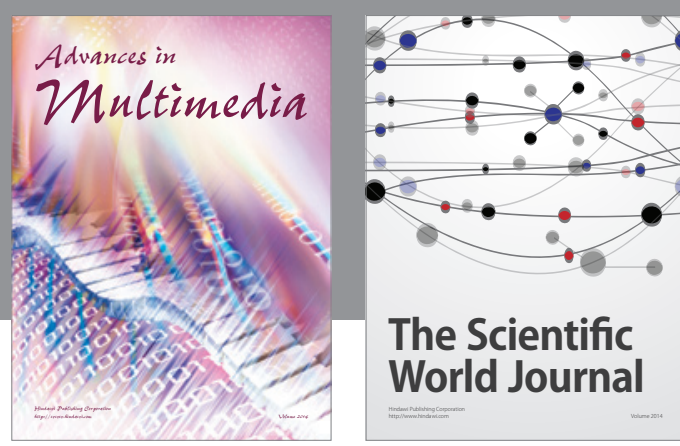

The Scientific World Journal
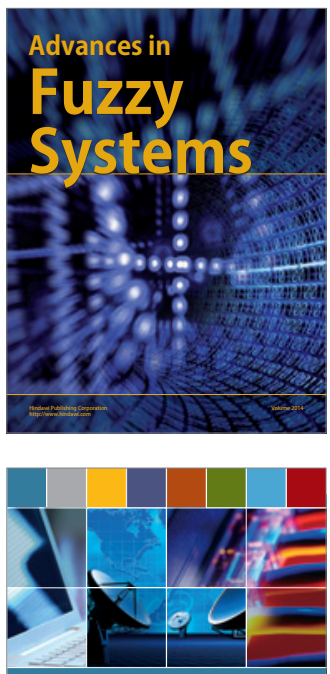

Computer Networks and Communications
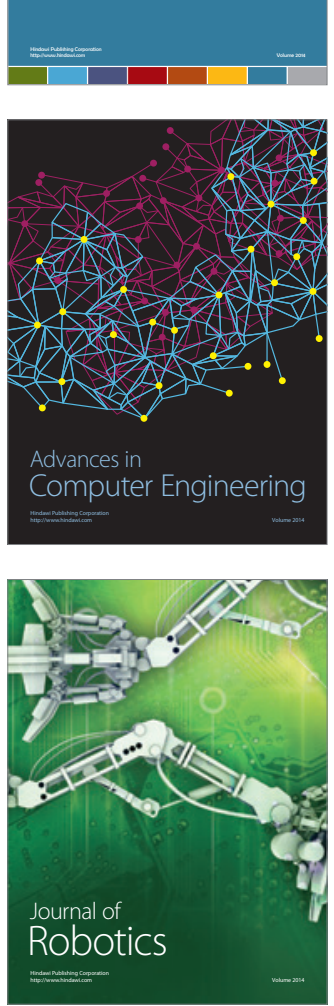
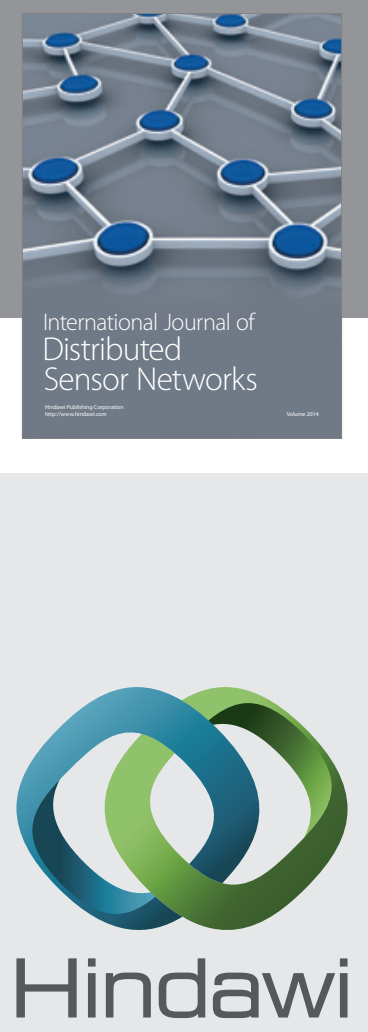

Submit your manuscripts at

http://www.hindawi.com
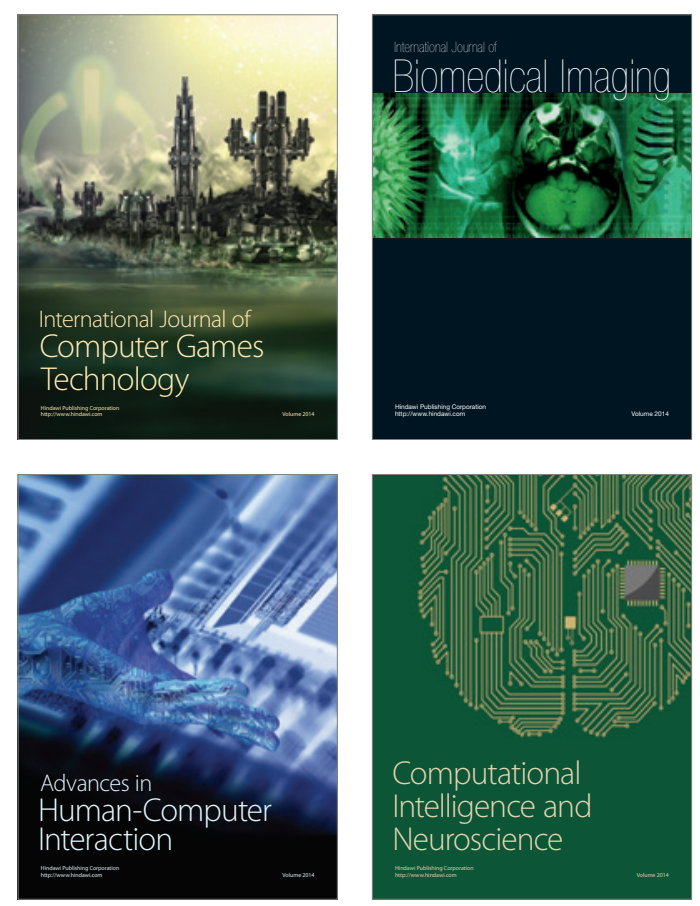
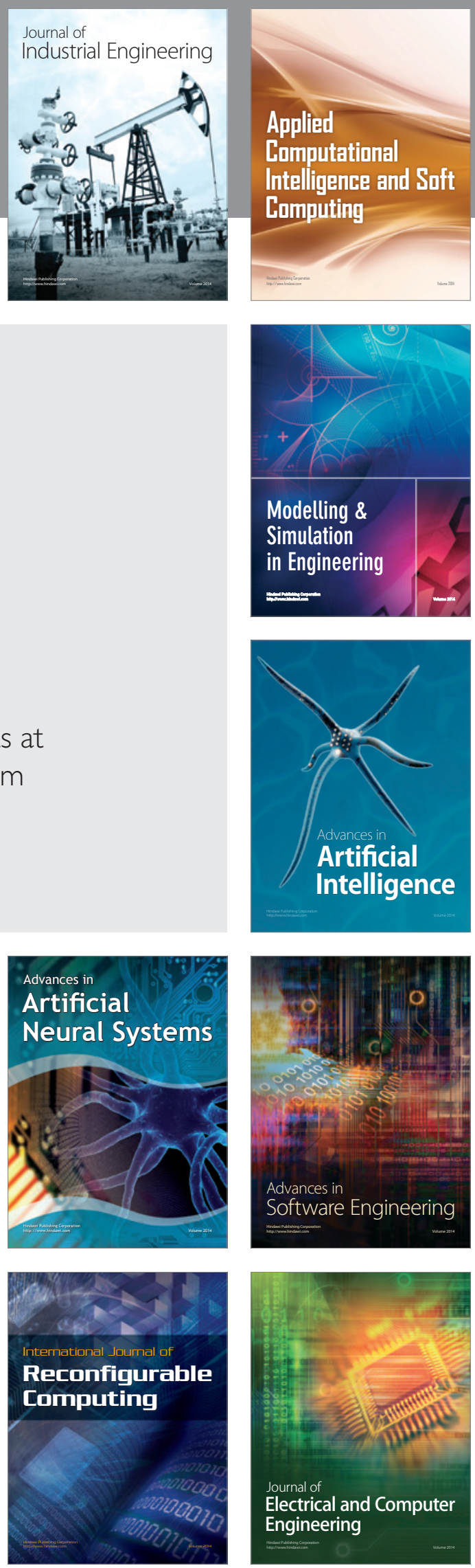